\title{
Thermal Relic Targets with Exponentially Small Couplings
}

\author{
Raffaele Tito D’Agnolo®, ${ }^{1, *}$ Duccio Pappadopulo๑, ${ }^{2, \dagger}$ Joshua T. Ruderman $\odot^{3, *}$ and Po-Jen Wang ${ }^{3, \S}$ \\ ${ }^{1}$ SLAC National Accelerator Laboratory, 2575 Sand Hill Road, Menlo Park, California 94025, USA \\ ${ }^{2}$ Bloomberg LP, New York, New York 10022, USA \\ ${ }^{3}$ Center for Cosmology and Particle Physics, Department of Physics, New York University, New York, New York 10003, USA
}

(Received 26 June 2019; revised manuscript received 16 January 2020; accepted 12 March 2020; published 17 April 2020)

\begin{abstract}
If dark matter was produced in the early Universe by the decoupling of its annihilations into known particles, there is a sharp experimental target for the size of its coupling. We show that if dark matter was produced by inelastic scattering against a lighter particle from the thermal bath, then its coupling can be exponentially smaller than the coupling required for its production from annihilations. As an application, we demonstrate that dark matter produced by inelastic scattering against electrons provides new thermal relic targets for direct detection and fixed target experiments.
\end{abstract}

DOI: $10.1103 /$ PhysRevLett.124.151801

Introduction.-Dark matter (DM) was produced in the early Universe by unknown dynamics. If the production of $\mathrm{DM}$ is tied to its measurable interactions, experiments can help disentangle its cosmological origin. The most studied example is the weakly interacting massive particle (WIMP) [1-4], with abundance set by the decoupling of its annihilations into standard model (SM) particles. In this case the DM annihilation rate is predicted and testable by current experiments.

A well-known variant of the WIMP is DM with a mass in the $\mathrm{MeV}$ to $\mathrm{GeV}$ range that annihilates into electrons and positrons (left of Fig. 1) [5,6]. This scenario requires additional force mediators beyond the SM [1,5-8]. The requirement that DM has the observed abundance fixes the size of its coupling to electrons, implying a sharp target for direct detection and fixed target experiments [9-14]. The WIMP and its variants are now driving a large experimental effort. Are there alternate scenarios on the same theoretical footing as the WIMP that experiments should also target?

In this Letter we consider thermal relic targets that share the attractive features of the WIMP. We seek cosmologies where DM (i) begins in thermal equilibrium with a temperature that tracks the photon temperature, (ii) follows a standard cosmological history, and (iii) has abundance determined by the decoupling of an interaction between DM and SM particles. The first two assumptions are violated by nonthermal DM [15-18], cannibalism [19-22], and late entropy production [23-25]; the third is not satisfied if DM

Published by the American Physical Society under the terms of the Creative Commons Attribution 4.0 International license. Further distribution of this work must maintain attribution to the author(s) and the published article's title, journal citation, and DOI. Funded by SCOAP . annihilates into hidden sector states $[6,26-30]$ or has abundance set by a primordial asymmetry [31-33].

We identify a new example that satisfies the above assumptions: DM, $\chi$, is produced by scattering against electrons. Elastic scattering, $\chi e^{ \pm} \rightarrow \chi e^{ \pm}$, does not change the number of DM particles, but the abundance can be set by inelastic scattering, $\chi e^{ \pm} \rightarrow \psi e^{ \pm}$(right of Fig. 1), where $\psi$ is a dark partner that experiences its own rapid annihilations. This is an example of coscattering [34] as studied recently by Refs. [35-39]. See also Refs. [40-42] for related prior work. Here we introduce a mechanism for extending any WIMP-like model to include a coscattering phase, and we show how this opens up new parameter space with smaller DM coupling.

The rate for DM to coscatter (annihilate) is proportional to the electron (DM) number density $n_{e}\left(n_{\chi}\right)$ multiplied by the relevant cross section. Suppose that $m_{e}<T_{f}<m_{\chi}$, where $T_{f}$ is the temperature at which annihilations or coscatterings decouple. Then the number density of electrons, which are relativistic, is larger than the number density of DM, which is nonrelativistic, by the exponentially large factor $n_{e} / n_{\chi} \propto e^{m_{\chi} / T_{f}}$. Therefore, the rate of coscattering can exceed annihilation despite an exponentially smaller cross

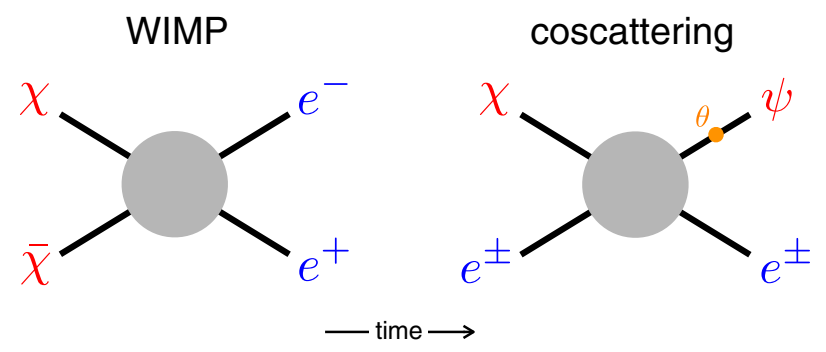

FIG. 1. The process setting the relic density for the WIMP (left) and coscattering (right), where $\chi$ is $\mathrm{DM}$ and $\psi$ is a dark partner. 
section, opening up vast new parameter space for thermal relic targets. Below we focus on a particular model with coscattering against electrons, but our observation applies to many more models and to replacing the electron with other SM particles. Another scenario that satisfies the above assumptions and leads to a smaller DM coupling is annihilation through a pole [43], requiring a special relation between the mass of DM and another particle.

The rest of this Letter is organized as follows. We begin by comparing coscattering to the more familiar WIMP, and we derive the exponential factor that enlarges the parameter space for coscattering. We then show how any model with annihilating DM can be extended to include coscattering, taking as an example scalar DM that couples to electrons through a dark photon. We explore the detailed phenomenology of this example, highlighting the experimentally testable parameter space. Our Supplemental Material [44] contains a map of the phase space of this model, charting where the DM abundance is set by coscattering or alternate mechanisms.

Coscattering vs annihilations.-A WIMP with mass $m_{\chi}$ begins in equilibrium with the SM thermal bath when $T>m_{\chi}$. Its abundance is depleted through annihilations when $T<m_{\chi}$. When the rate of DM annihilations becomes slower than the expansion of the Universe,

$$
n_{\chi}^{\mathrm{eq}}\langle\sigma v\rangle \approx H,
$$

the total number of DM particles is fixed (freezes-out) determining the observed relic density today $[2,3]$. Here we have used the superscript "eq" to denote particles in thermal equilibrium with the SM bath. The sudden freeze-out approximation described above points to annihilation cross sections comparable to those induced by the SM weak interactions:

$$
\frac{\Omega_{\mathrm{WIMP}}}{\Omega_{\mathrm{DM}}} \approx \frac{0.3 \mathrm{pb}}{\langle\sigma v\rangle} \frac{x_{f} \sqrt{g_{*}}}{g_{* S}} \equiv \frac{\sigma_{\mathrm{WIMP}}}{\langle\sigma v\rangle},
$$

where $x_{f}=T_{f} / m_{\chi}$ describes the temperature when Eq. (1) is satisfied.

If annihilations are too small, and no other process removes DM, the relic density is too large. In this regime, coscattering is an alternative mechanism for setting the DM abundance [34]. DM still begins in equilibrium with the SM thermal bath when $T>m_{\chi}$. The relic density is set when $T<m_{\chi}$ and inelastic scattering becomes slower than the Hubble expansion rate. In the following we assume that DM scatters against an electron or positron, as on the right of Fig. 1, but other choices are possible [34]. DM scatters into a dark partner $\psi$ with a heavier mass, $m_{\psi} \geq m_{\chi}$, whose annihilations leave equilibrium after the inelastic scattering. $\mathrm{DM}$ is depleted by $\chi e^{ \pm} \rightarrow \psi e^{ \pm}$followed by $\psi$ annihilations. When $\chi e^{ \pm} \rightarrow \psi e^{ \pm}$freezes-out, so does the $\chi$ abundance. The small component of $\psi$ left over from the later freeze-out of $\psi$ annihilations is converted into $\chi$ by decays, or else persists until today.

The main qualitative difference between coscattering and the WIMP is that we can obtain the correct relic density with $\chi$ interactions that are exponentially smaller than required for annihilations. If we assume that momentum transfer via $\chi e^{ \pm} \rightarrow \chi e^{ \pm}$is efficient until after the coscattering diagram freezes-out, we can write a Boltzmann equation for the total $\chi$ number density

$$
\frac{d n_{\chi}}{d t}+3 H n_{\chi}=-n_{e}^{\mathrm{eq}}\left\langle\sigma_{\chi \rightarrow \psi} v\right\rangle\left[n_{\chi}-n_{\chi}^{\mathrm{eq}}\right] .
$$

In this case freeze-out occurs when

$$
n_{e}^{\mathrm{eq}}\left\langle\sigma_{\chi \rightarrow \psi} v\right\rangle \approx p H
$$

where $p \approx 10$ is chosen to match numerical solutions to Eq. (3). If we use detailed balance to write the thermal average for the endothermic process $\chi \rightarrow \psi$ in terms of the thermal average for the inverse process [34], we can write the $\chi$ relic density as

$$
\frac{\Omega_{\chi}}{\Omega_{\mathrm{DM}}} \approx 0.2 \frac{\sigma_{\mathrm{WIMP}}}{\left\langle\sigma_{\psi \rightarrow \chi} v\right\rangle} \frac{p g_{\chi}^{2} x_{f}^{3 / 2} e^{-x_{f}\left(1-\frac{\Delta m}{m_{\chi}}\right)}}{g_{\psi}\left(1+\Delta m / m_{\chi}\right)^{3 / 2}},
$$

where $\Delta m \equiv m_{\psi}-m_{\chi}$ and $g_{\chi, \psi}$ counts the number of degrees of freedom of $\chi$ and $\psi$. In order to reproduce $\Omega_{\chi}=$ $\Omega_{\mathrm{DM}}$ we require $\left\langle\sigma_{\psi \rightarrow \chi} v\right\rangle \sim e^{-x_{f}\left(1-\Delta m / m_{\chi}\right)} \sigma_{\mathrm{WIMP}}$. This corresponds to a significant exponential suppression, as long as $\Delta m / m_{\chi}<1$, because $x_{f} \sim 20$ is needed to match the observed abundance. (The necessary freeze-out temperature is universal across different types of thermal relics including coscattering and the WIMP).

The model. - The coscattering phase can be added to any WIMP model in a modular way. Consider a WIMP with an annihilation channel, such as the left of Fig. 1, and add a mass mixing between DM and a dark partner $\psi$ with its own rapid annihilations. The coscattering diagram on the right of Fig. 1 is generated by rotating the annihilation diagram and inserting the mixing.

As an example we consider dark scalar QED [5,6,9-14] with DM coupled to the SM via a $U(1)_{d}$ massive dark photon, $A_{d}$, kinetically mixed [47] with the ordinary photon, $A$ :

$$
\mathcal{L} \supset-\frac{1}{4} F_{\mu \nu}^{2}-\frac{1}{4}\left(F_{d}^{\mu \nu}\right)^{2}-\frac{\epsilon}{2} F_{d}^{\mu \nu} F_{\mu \nu}-\frac{m_{A}^{2}}{2} A_{d}^{2},
$$

where $F_{(d)}$ is the ordinary (dark) photon field strength, and $\epsilon$ is a dimensionless measure of the mixing. DM is a complex scalar $\chi$ with charge 1 under $U(1)_{d}: \mathcal{L} \supset\left|D_{\mu} \chi\right|^{2}-\bar{m}_{\chi}^{2}|\chi|^{2}$. DM can annihilate $\chi \chi^{*} \rightarrow \gamma_{d}^{*} \rightarrow e^{+} e^{-}$. 

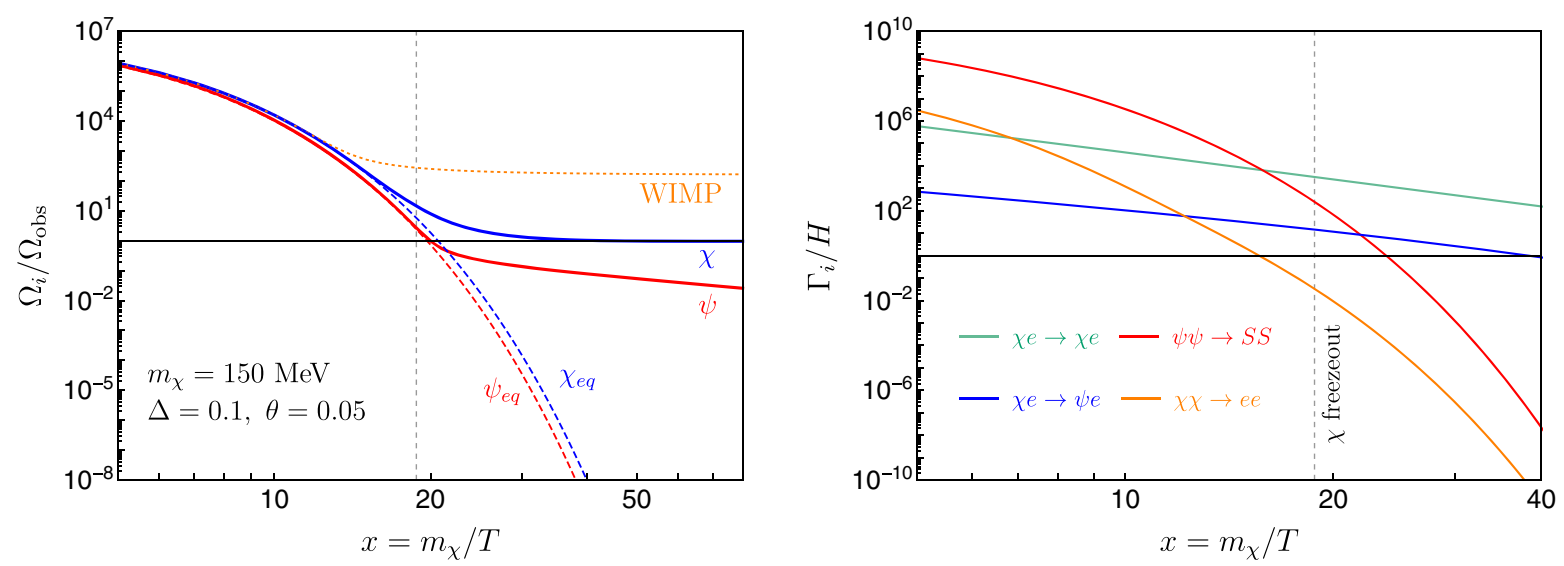

FIG. 2. Left panel: Evolution of the DM energy density for an ordinary WIMP (orange dotted) and coscattering (solid blue). The red curve describes freeze-out of the DM partner $\psi$. Right panel: thermal rates of the interactions relevant for coscattering as a function of $x=m_{\chi} / T$. We plot the momentum transfer rate for elastic scattering (green) and $\left(n_{\psi}^{\mathrm{eq}}\right)^{2}\langle\sigma v\rangle / n_{\chi}^{\mathrm{eq}}$ for $\psi$ annihilations (red). In both plots, $m_{\chi}=150 \mathrm{MeV}, \Delta=0.1, \theta=0.05, \epsilon=3 \times 10^{-5}, \lambda_{\psi S}=0.0064, \alpha_{d}=0.5, m_{S}=156 \mathrm{MeV}$, and $m_{A}=3 m_{\chi}$.

We add a complex scalar $\psi$ that is neutral under $U(1)_{d}$ and mixed with $\chi$ :

$$
\mathcal{L} \supset\left|\partial_{\mu} \psi\right|^{2}-\bar{m}_{\psi}^{2}|\psi|^{2}-\delta m^{2}\left(\chi^{*} \psi+\text { H.c. }\right) \text {. }
$$

Coscattering $\chi e \rightarrow \psi e$ is generated by the mixing. Quartic couplings for $\chi$ and $\psi$ can also be included without modifying our discussion. The mass mixing $\delta m^{2}$ and the dark photon mass $m_{A}^{2}$ can arise from a dark Higgs coupled to $\chi$ and $\psi$. Note that we use $\bar{m}_{\chi, \psi}^{2}$ for mass parameters in the Lagrangian and $m_{\chi, \psi}^{2}$ for mass eigenvalues. The angle that rotates from the Lagrangian basis to the mass eigenstate basis is $\theta \sim \delta m^{2} / m_{\chi}^{2}$. For the rest of this Letter we (slightly) abuse notation by calling the lightest eigenstate $\chi$ and the heaviest $\psi$.

We take $\psi$ to annihilate to a dark state (this is a key difference versus Refs. [35,37,38], where the dark partner has a large coupling to the SM), $\psi \psi^{*} \rightarrow S S$, where $S$ is a real scalar with mass $m_{S}<m_{\psi}$ and interactions

$$
\mathcal{L} \supset-\frac{\lambda_{\psi S}}{2} S^{2}|\psi|^{2}-y_{e S}\left(S e^{c} e+\text { H.c. }\right)
$$

$\chi$ couples to $S$ only through its mixing with $\psi$. We take $S$ to decay rapidly to the thermal bath via a small Yukawa coupling to electrons. There is a large range of values for this coupling $\left(10^{-9} \lesssim y_{e S} \lesssim 10^{-4}\right)$ such that $S$ remains in equilibrium with the SM bath during freeze-out but otherwise $y_{e S}$ does not enter the relic density calculation. Above the scale of electroweak symmetry breaking, this Yukawa can be generated by the effective operator $y_{e}(S / \Lambda) l H e^{c}$, where $y_{e}$ is the SM electron Yukawa coupling. For an example ultraviolet completion see Ref. [48], where $y_{e S} \lesssim 10^{-7}$ is consistent with other constraints for the range of $m_{S}$ that we consider. We stress that $S \rightarrow e^{+} e^{-}$is just one possibility, the following discussion applies for any rapid $S$ decays including to photons or dark radiation.

We consider parameters where $\chi \chi^{*} \rightarrow e^{+} e^{-}$annihilations are too feeble to set the relic density. However $\psi \psi^{*} \rightarrow S S$ annihilations are efficient and the last process to freeze-out. The coscattering process $\chi e \rightarrow \psi e$ leaves equilibrium after $\chi \chi^{*} \rightarrow e^{+} e^{-}$, but before $\psi \psi^{*} \rightarrow S S$. It is the fastest process converting $\chi$ to $\psi$ and its freeze-out determines the $\chi$ abundance. This hierarchy of thermal rates is depicted in Fig. 2. The figure also shows that $\chi$ would have too large of an abundance in the WIMP limit where only $\chi$ annihilations are active, while the addition of $\psi$ leads to the correct relic density via coscattering.

The freeze-out hierarchy described in the previous paragraph can be realized with the spectrum: $m_{A}>m_{\psi}>$ $m_{S}>m_{\chi} \gtrsim \sqrt{\delta m^{2}}$ and the hierarchy of couplings: $g_{d}=$ $\mathcal{O}(1) \gg \epsilon, \lambda_{\psi S}$. Furthermore, we fix $r_{S} \equiv\left(m_{S}-m_{\chi}\right) /$ $\left(m_{\psi}-m_{\chi}\right)=0.75$ in the following. More details on these choices and the freeze-out phases of this theory can be found in the Supplemental Material [44].

Phenomenology.-The main qualitative feature of coscattering with SM states is the exponentially small coupling to SM particles, compared to the WIMPIn Fig. 3 we plot current constraints and future probes in terms of the scattering cross section relevant to electron recoil experiments: $\bar{\sigma}_{e} \approx e^{2} g_{d}^{2} \epsilon^{2} m_{e}^{2} \cos ^{4} \theta /\left(\pi m_{A}^{4}\right)$. Coscattering can reproduce the observed relic density in the region between the solid orange line (where it reduces to WIMP freeze-out) and the solid blue line. In the coscattering region we find that $\chi$ is in kinetic equilibrium with the SM during freeze-out due to rapid energy exchange from $\chi e^{ \pm} \rightarrow \chi e^{ \pm}[49,50]$. This is distinct from previous studies of coscattering where DM kinetically decouples during freeze-out [34-38]. 


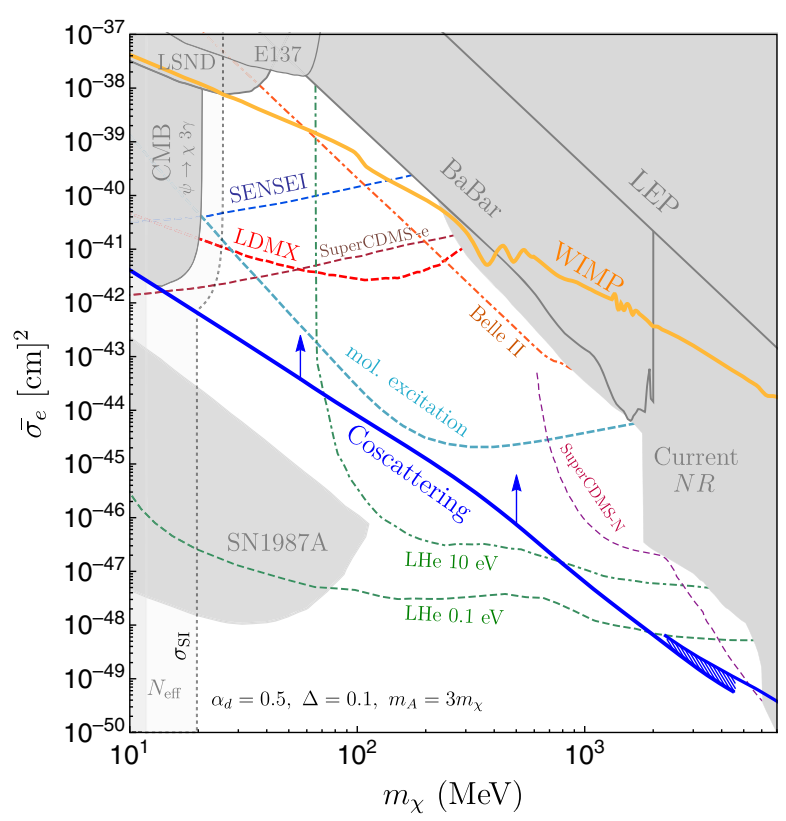

FIG. 3. Existing constraints and reach of future experiments as a function of the DM mass $m_{\chi}$, expressed in terms of the direct detection cross section for electron recoils $\bar{\sigma}_{e}$. In the region between the orange and the blue lines the observed relic density is obtained via the coscattering mechanism. In the plot we fix $\alpha_{d}=0.5, m_{A}=3 m_{\chi}, r_{S}=0.75$, and $\Delta \equiv\left(m_{\psi}^{2}-m_{\chi}^{2}\right) / m_{\chi}^{2}=0.1$.

Proposed experiments have the potential to probe a large fraction of the coscattering region, as shown in Fig. 3. They include electron recoil direct detection experiments such as SENSEI [13,51-53] (shown for $100 \mathrm{~g}$-yr exposure) and SuperCDMS-G2+ [13] (1 kg-yr); nucleon recoil direct detection including SuperCDMS iZIP detectors [13] ( $\mathrm{Si}$, $4.8 \mathrm{~kg}-\mathrm{yr}$ ), molecular excitations [54] (250 gr-yr), and superfluid helium detectors [55] (1 kg-yr with a $10 \mathrm{eV}$ energy threshold and $10 \mathrm{~kg}-\mathrm{yr}$ with $0.1 \mathrm{eV}$ ); missing momentum fixed target experiments such as LDMX [14,56]; and the Belle-II high-luminosity electron-positron

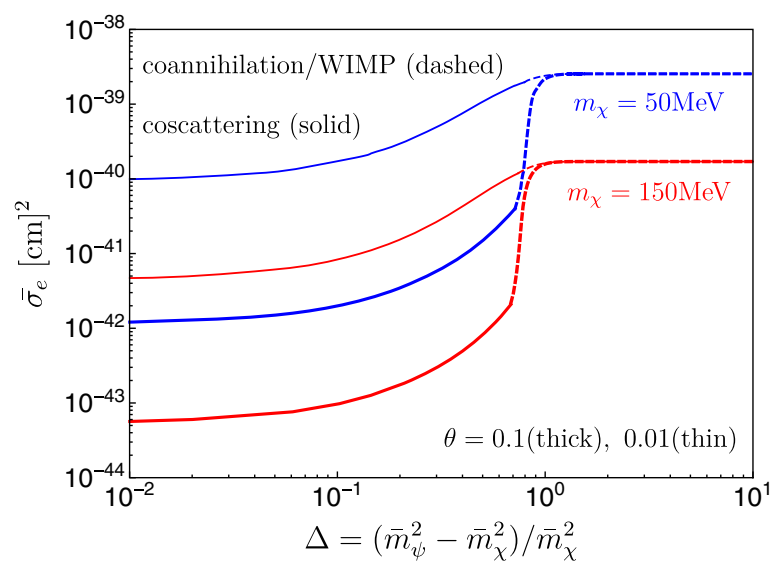

collider [57]. We also note that NA64 [58] and other proposed direct detection experiments [13] have potential sensitivity to our parameter space.

In Fig. 3 we fix $\alpha_{d}=0.5, m_{A}=3 m_{\gamma}, r_{S}=0.75$, and $\Delta \equiv\left(m_{\psi}^{2}-m_{\chi}^{2}\right) / m_{\chi}^{2}=0.1$. Within the coscattering region $\epsilon$ is determined by the value of $\bar{\sigma}_{e}$ on the $y$ axis, once $\theta$ is fixed at each point to give the right relic density. In this region the quartic $\lambda_{\psi S}$ is chosen at each point to select the coscattering regime, as discussed in Supplemental Material [44]. Below the coscattering region the mixing angle is set to its maximum value $\theta=0.45$ that is reached on the blue line at the boundary of the coscattering parameter space.

The reason why coscattering provides a range of viable couplings as opposed to the WIMP relic density line can be understood with the help of Fig. 4. The relic density is set by processes that interchange $\chi$ and $\psi$, so is sensitive to the mixing angle $\theta$ as shown in the right panel of Fig. 4. Larger $\theta$ allows for a smaller coupling of $\chi$ to electrons when fixing the relic density. So the smallest possible coupling of $\mathrm{DM}$ to the $\mathrm{SM}$ is achieved when $\theta=\mathcal{O}(1)$. Smaller values of $\theta$ span the region between the blue and the orange lines of Fig. 3. When $\theta$ becomes too large we enter a coannihilation or WIMP regime, depending on the parameters in the dark sector, as shown in Fig. 4 and discussed in the Supplemental Material [44].

We also comment on the $\chi-\psi$ mass splitting, $\Delta$. The coscattering process is endothermic $\left(m_{\psi} \geq m_{\chi}\right)$ and the relic density depends exponentially on $\Delta$. This is shown in the left panel of Fig. 4 and in Eq. (5). When $\Delta$ goes to zero there is no suppression from the thermal average and $\bar{\sigma}_{e}$ can be small, while for larger $\Delta$ a larger coupling of $\chi$ to electrons has to compensate the kinematical suppression. So $\Delta \approx 0$ gives the smallest direct detection cross section compatible with the observed relic density. In Fig. 3 we have chosen $\Delta=0.1$; the smallest possible $\bar{\sigma}_{e}$ obtained for $\Delta=0$ is approximately a factor of 5 below the solid blue line in the plot.

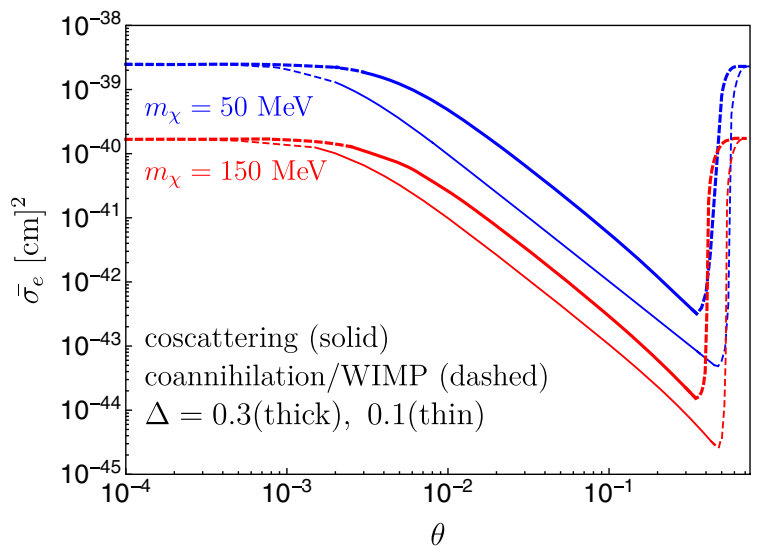

FIG. 4. Direct detection cross section for scattering off atomic electrons $\bar{\sigma}_{e}$ as a function of the mass splitting between DM and its partner $\psi$ (left panel) and their mixing (right panel), after the DM relic density is fixed to its observed value. In both plots we fix $\alpha_{d}=0.5, m_{A}=3 m_{\chi}$, and $r_{S}=0.75$. 
We would like to draw attention to the relative smoothness of the line bounding the coscattering parameter space from below in Fig. 3, compared to the WIMP relic density line. The WIMP line is determined by $s$-channel DM annihilations and reflects the structure of SM resonances that occur when $2 m_{\chi} \approx m_{\mathrm{SM}}$. Coscattering is $t$ channel and receives contributions from all SM states that are relativistic at freezeout, $T_{f} \gtrsim m_{\mathrm{SM}}$. As the DM mass increases, new SM states contribute smoothly. There is theoretical uncertainty when freeze-out happens near the QCD phase transition, $T_{f} \sim 100-200 \mathrm{MeV}$. In this regime we show both coscattering off quarks (upper curve) and pions or kaons (lower curve).

In Fig. 3 we also show current constraints on our parameter space (shaded in gray). We display CMB bounds on $\psi \rightarrow \chi+3 \gamma$ decays [59] and $N_{\text {eff }}$ [60]; supernova cooling [61]; direct detection from nuclear recoils [62-66]; BABAR missing energy searches [67]; LEP measurements at the $Z$ pole [68]; beam dump experiments such as LSND [69,70] and E137 [71,72]; and DM self-interactions (we require $\sigma_{\mathrm{SI}} / m_{\chi} \gtrsim 1 \mathrm{~cm}^{2} / \mathrm{g}$ ) [73-78].

Conclusions. - In this Letter we introduced a thermal relic that shares the attractive theoretical properties of the WIMP, but reproduces the DM relic density with an exponentially smaller coupling to the SM. We have shown that coscattering [34] can be realized by extending any model of WIMP DM, opening up orders of magnitude of new parameter space. We have explored the phenomenology of one concrete example model, providing a new benchmark for future light DM experiments. Our work provides further motivation for direct detection experiments that probe sub-GeV DM scattering with nucleons or electrons, and missing momentum searches at fixed target experiments. Coscattering motivates extending the sensitivity of these programs beyond traditional WIMP targets.

We would like to thank A. Berlin, P. Schuster, and N. Toro for useful discussions. R. T. D. is supported by DOE Grant No. DE-AC02-76SF00515. J. T. R. is supported by NSF CAREER Grant No. PHY-1554858 and NSF grant No. PHY-1915409. R. T. D. acknowledges support by the Munich Institute for Astro- and Particle Physics (MIAPP) which is funded by the Deutsche Forschungsgemeinschaft (DFG, German Research Foundation) under Germany's Excellence Strategy EXC-2094-390783311. R. T. D. acknowledges support by KITP, through the National Science Foundation under Grant No. NSF PHY-1748958. J. T. R. acknowledges hospitality from the Aspen Center for Physics, which is supported by NSF Grant No. PHY-1607611.

*dagnolo@slac.stanford.edu

†duccio.pappadopulo@gmail.com

*uderman@nyu.edu

§pjw319@nyu.edu

[1] B. W. Lee and S. Weinberg, Phys. Rev. Lett. 39, 165 (1977).
[2] E. W. Kolb and M. S. Turner, The Early Universe (1990), Vol. 69, pp. 1-547, https://inspirehep.net/literature/299778.

[3] P. Gondolo and G. Gelmini, Nucl. Phys. B360, 145 (1991).

[4] G. Jungman, M. Kamionkowski, and K. Griest, Phys. Rep. 267, 195 (1996).

[5] C. Boehm and P. Fayet, Nucl. Phys. B683, 219 (2004).

[6] M. Pospelov, A. Ritz, and M. B. Voloshin, Phys. Lett. B 662, 53 (2008).

[7] M. J. Strassler and K. M. Zurek, Phys. Lett. B 651, 374 (2007).

[8] N. Arkani-Hamed, D. P. Finkbeiner, T. R. Slatyer, and N. Weiner, Phys. Rev. D 79, 015014 (2009).

[9] E. Izaguirre, G. Krnjaic, P. Schuster, and N. Toro, Phys. Rev. D 91, 094026 (2015).

[10] E. Izaguirre, G. Krnjaic, P. Schuster, and N. Toro, Phys. Rev. Lett. 115, 251301 (2015).

[11] R. Essig, M. Fernandez-Serra, J. Mardon, A. Soto, T. Volansky, and T.-T. Yu, J. High Energy Phys. 05 (2016) 046.

[12] J. Alexander et al., arXiv:1608.08632.

[13] M. Battaglieri et al., in U.S. Cosmic Visions: New Ideas in Dark Matter College Park, MD, USA, 2017 (2017) [arXiv:1707.04591].

[14] A. Berlin, N. Blinov, G. Krnjaic, P. Schuster, and N. Toro, Phys. Rev. D 99, 075001 (2019).

[15] T. Moroi, H. Murayama, and M. Yamaguchi, Phys. Lett. B 303, 289 (1993).

[16] S. Dodelson and L. M. Widrow, Phys. Rev. Lett. 72, 17 (1994).

[17] J. L. Feng, A. Rajaraman, and F. Takayama, Phys. Rev. Lett. 91, 011302 (2003).

[18] L. J. Hall, K. Jedamzik, J. March-Russell, and S. M. West, J. High Energy Phys. 03 (2010) 080.

[19] E. D. Carlson, M. E. Machacek, and L. J. Hall, Astrophys. J. 398, 43 (1992).

[20] E. Kuflik, M. Perelstein, N. Rey-Le Lorier, and Y.-D. Tsai, Phys. Rev. Lett. 116, 221302 (2016).

[21] D. Pappadopulo, J. T. Ruderman, and G. Trevisan, Phys. Rev. D 94, 035005 (2016).

[22] M. Farina, D. Pappadopulo, J. T. Ruderman, and G. Trevisan, J. High Energy Phys. 12 (2016) 039.

[23] G. B. Gelmini and P. Gondolo, Phys. Rev. D 74, 023510 (2006).

[24] C. Wainwright and S. Profumo, Phys. Rev. D 80, 103517 (2009).

[25] A. Berlin, D. Hooper, and G. Krnjaic, Phys. Lett. B 760, 106 (2016).

[26] D. P. Finkbeiner and N. Weiner, Phys. Rev. D 76, 083519 (2007).

[27] J. L. Feng and J. Kumar, Phys. Rev. Lett. 101, 231301 (2008).

[28] Y. Hochberg, E. Kuflik, T. Volansky, and J. G. Wacker, Phys. Rev. Lett. 113, 171301 (2014).

[29] Y. Hochberg, E. Kuflik, H. Murayama, T. Volansky, and J. G. Wacker, Phys. Rev. Lett. 115, 021301 (2015).

[30] J. A. Evans, S. Gori, and J. Shelton, J. High Energy Phys. 02 (2018) 100.

[31] D. E. Kaplan, M. A. Luty, and K. M. Zurek, Phys. Rev. D 79, 115016 (2009).

[32] K. Petraki and R. R. Volkas, Int. J. Mod. Phys. A 28, 1330028 (2013).

[33] K. M. Zurek, Phys. Rep. 537, 91 (2014). 
[34] R. T. D’Agnolo, D. Pappadopulo, and J. T. Ruderman, Phys. Rev. Lett. 119, 061102 (2017).

[35] M. Garny, J. Heisig, B. Lulf, and S. Vogl, Phys. Rev. D 96, 103521 (2017).

[36] H.-C. Cheng, L. Li, and R. Zheng, J. High Energy Phys. 09 (2018) 098.

[37] M. Garny, J. Heisig, M. Hufnagel, and B. Lulf, Phys. Rev. D 97, 075002 (2018).

[38] M. Garny, J. Heisig, M. Hufnagel, B. Lulf, and S. Vogl, Proc. Sci. CORFU2018 (2019) 092.

[39] H. Kim and E. Kuflik, Phys. Rev. Lett. 123, 191801 (2019).

[40] G. R. Farrar and E. W. Kolb, Phys. Rev. D 53, 2990 (1996).

[41] D. J. H. Chung, G. R. Farrar, and E. W. Kolb, Phys. Rev. D 56, 6096 (1997).

[42] M. Pospelov and J. Pradler, Phys. Rev. D 82, 103514 (2010).

[43] J. L. Feng and J. Smolinsky, Phys. Rev. D 96, 095022 (2017).

[44] See Supplemental Material at http://link.aps.org/supplemental/ 10.1103/PhysRevLett.124.151801 for an analytical understanding of the phase diagram of our model. This Supplemental Material includes Refs. [14,34,45,46].

[45] R. T. D’Agnolo, C. Mondino, J. T. Ruderman, and P.-J. Wang, J. High Energy Phys. 08 (2018) 079.

[46] K. Griest and D. Seckel, Phys. Rev. D 43, 3191 (1991).

[47] B. Holdom, Phys. Lett. 166B, 196 (1986).

[48] B. Batell, N. Lange, D. McKeen, M. Pospelov, and A. Ritz, Phys. Rev. D 95, 075003 (2017).

[49] T. Bringmann and S. Hofmann, J. Cosmol. Astropart. Phys. 04 (2007) 016; 03 (2016) E02.

[50] P. Adshead, Y. Cui, and J. Shelton, J. High Energy Phys. 06 (2016) 016.

[51] J. Tiffenberg, M. Sofo-Haro, A. Drlica-Wagner, R. Essig, Y. Guardincerri, S. Holland, T. Volansky, and T.-T. Yu (SENSEI Collaboration), Phys. Rev. Lett. 119, 131802 (2017).

[52] O. Abramoff et al. (SENSEI Collaboration), Phys. Rev. Lett. 122, 161801 (2019).

[53] M. Crisler, R. Essig, J. Estrada, G. Fernandez, J. Tiffenberg, M. S. Haro, T. Volansky, and T.-T. Yu (SENSEI Collaboration), Phys. Rev. Lett. 121, 061803 (2018).

[54] R. Essig, J. Pérez-Ríos, H. Ramani, and O. Slone, Phys. Rev. Research 1, 033105 (2019).

[55] S. A. Hertel, A. Biekert, J. Lin, V. Velan, and D. N. McKinsey, Phys. Rev. D 100, 092007 (2019).

[56] T. A kesson, arXiv:1808.05219.
[57] W. Altmannshofer et al. (Belle-II Collaboration), Prog. Theor. Exp. Phys. 2019, 123C01 (2019).

[58] S. N. Gninenko, D. V. Kirpichnikov, M. M. Kirsanov, and N. V. Krasnikov, Phys. Lett. B 796, 117 (2019).

[59] T. R. Slatyer and C.-L. Wu, Phys. Rev. D 95, 023010 (2017).

[60] N. Aghanim et al. (Planck Collaboration), arXiv:1807.06209.

[61] J. H. Chang, R. Essig, and S. D. McDermott, J. High Energy Phys. 09 (2018) 051.

[62] A. H. Abdelhameed et al. (CRESST Collaboration), Phys. Rev. D 100, 102002 (2019).

[63] R. Agnese et al. (SuperCDMS Collaboration), Phys. Rev. Lett. 116, 071301 (2016).

[64] P. Agnes et al. (DarkSide Collaboration), Phys. Rev. Lett. 121, 081307 (2018).

[65] X. Cui et al. (PandaX-II Collaboration), Phys. Rev. Lett. 119, 181302 (2017).

[66] E. Aprile et al. (XENON Collaboration), Phys. Rev. Lett. 121, 111302 (2018).

[67] J. P. Lees et al. (BABAR Collaboration), Phys. Rev. Lett. 119, 131804 (2017).

[68] A. Hook, E. Izaguirre, and J. G. Wacker, Adv. High Energy Phys. 2011, 859762 (2011).

[69] L. B. Auerbach et al. (LSND Collaboration), Phys. Rev. D 63, 112001 (2001).

[70] P. deNiverville, M. Pospelov, and A. Ritz, Phys. Rev. D 84, 075020 (2011).

[71] J. D. Bjorken, S. Ecklund, W. R. Nelson, A. Abashian, C. Church, B. Lu, L.W. Mo, T. A. Nunamaker, and P. Rassmann, Phys. Rev. D 38, 3375 (1988).

[72] B. Batell, R. Essig, and Z. Surujon, Phys. Rev. Lett. 113, 171802 (2014).

[73] M. Rocha, A. H. G. Peter, J. S. Bullock, M. Kaplinghat, S. Garrison-Kimmel, J. Onorbe, and L. A. Moustakas, Mon. Not. R. Astron. Soc. 430, 81 (2013).

[74] A. H. G. Peter, M. Rocha, J. S. Bullock, and M. Kaplinghat, Mon. Not. R. Astron. Soc. 430, 105 (2013).

[75] M. Markevitch, A. H. Gonzalez, D. Clowe, A. Vikhlinin, L. David, W. Forman, C. Jones, S. Murray, and W. Tucker, Astrophys. J. 606, 819 (2004).

[76] D. Clowe, A. Gonzalez, and M. Markevitch, Astrophys. J. 604, 596 (2004).

[77] S. W. Randall, M. Markevitch, D. Clowe, A. H. Gonzalez, and M. Bradac, Astrophys. J. 679, 1173 (2008).

[78] D. Harvey, R. Massey, T. Kitching, A. Taylor, and E. Tittley, Science 347, 1462 (2015). 